No potential conflict of interest relevant to this letter was reported.

1. DeFronzo RA, Abdul-Ghani M. Assessment and treatment of cardiovascular risk in prediabetes: impaired glucose tolerance and impaired fasting glucose. Am J Cardiol 2011;108:Suppl:3B24B.

2. Sjöström L, Peltonen $M$, Jacobson $P$, et al. Bariatric surgery and long-term cardiovascular events. JAMA 2012;307:56-65.

3. Després JP. What is "metabolically healthy obesity"?: from epidemiology to pathophysiological insights. J Clin Endocrinol Metab 2012;97:2283-5.

4. Tschöp MH, DiMarchi RD. Outstanding Scientific Achievement Award Lecture 2011: defeating diabesity: the case for personalized combinatorial therapies. Diabetes 2012;61:1309-14.

DOI: $10.1056 / \mathrm{NEJMc1} 211380$

THE AUTHORS REPLY: We are aware of the new diabetes criteria mentioned by Barajas-Gamboa and Diaz-Perez. However, glycated hemoglobin level was not typically included in the diagnosis of diabetes when the SOS study started in 1987, and glucose-tolerance testing at more than 500 study sites was not logistically achievable. We carefully specified the criteria used for diabetes diagnosis in our article, and the fact that they are not in agreement with the latest definitions was also pointed out as a limitation. We have no reason to believe that slightly different criteria would have changed our conclusion that bariatric surgery prevents diabetes.

In response to Zaccardi et al.: we would point out that there are no real conflicts between their statements and our findings. In the SOS study population (BMI $\geq 34$ ), we have found no interactions between BMI and treatment (or between other anthropometric variables and treatment) with respect to the incidence of diabetes, ${ }^{1}$ cardiovascular events, ${ }^{2}$ or cancer ${ }^{3}$ or with respect to overall mortality, ${ }^{4}$ whereas glucose and insulin levels were predictors of treatment effect for several of these end points. We agree that reduced insulin resistance may be one of the mechanisms behind the effects of bariatric surgery, and for cardiovascular events, the baseline insulin level was the only predictor (among 20 variables tested) of the surgical treatment effect. ${ }^{2}$ Subgroup-treatment interactions with respect to hard end points are, to the best of our knowledge, not available in other studies of bariatric surgery.

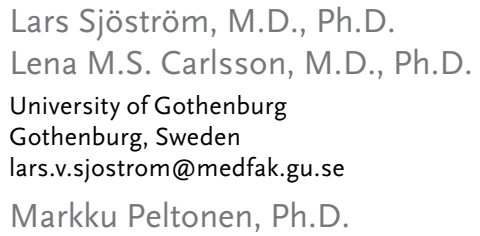

National Institute for Health and Welfare Helsinki, Finland

Since publication of their article, the authors report no further potential conflict of interest.

1. Sjöström L, Lindroos AK, Peltonen M, et al. Lifestyle, diabetes and cardiovascular risk factors 10 years after bariatric surgery. N Engl J Med 2004;351:2683-93.

2. Sjöström L, Peltonen $M$, Jacobson $P$, et al. Bariatric surgery and long-term cardiovascular events. JAMA 2012;307:56-65.

3. Sjöström L, Gummesson A, Sjöström D, et al. Effects of bariatric surgery on cancer incidence in obese patients in Sweden (Swedish Obese Subjects Study): a prospective, controlled intervention trial. Lancet Oncol 2009;10:653-62.

4. Sjöström L, Narbro K, Sjöström CD, et al. Effects of bariatric surgery on mortality in Swedish obese subjects. N Engl J Med 2007;357:741-52.

DOI: 10.1056/NEJMc1211380

\title{
Antibiotic Prevention of Acute Exacerbations of COPD
}

TO THE EDITOR: Wenzel and colleagues (July 26 issue $)^{1}$ recommend long-term azithromycin for a 55-year-old man with recurrent exacerbations of chronic obstructive pulmonary disease (COPD), a forced expiratory volume in 1 second $\left(\mathrm{FEV}_{1}\right)$ of $50 \%$ of the predicted value, dyspnea, cough, and sputum production. However, before azithromycin, we think this patient should be treated with other pharmacologic agents to manage his COPD and prevent exacerbations.

Tiotropium is a long-acting anticholinergic bronchodilator that improves the $\mathrm{FEV}_{1}$ and healthrelated quality of life. Tiotropium reduces the frequency of exacerbations and related hospitalizations. More important, tiotropium is effective even when patients are receiving inhaled glucocorticoids and long-acting beta-agonists. ${ }^{2}$

This patient's history of cough and sputum production suggests chronic bronchitis. The phosphodiesterase-4 inhibitor roflumilast reduces moderate-to-severe exacerbations in patients with chronic bronchitis, severe-to-very-severe COPD, and a history of exacerbations. Favorable effects are also seen when roflumilast is added to long-acting bronchodilators. ${ }^{3}$

In our view, before recommending azithro- 
mycin, this patient should be treated with tiotropium. Roflumilast should be added if he continues to have exacerbations. Azithromycin should be considered only if the exacerbations are not adequately controlled despite the addition of tiotropium and roflumilast.

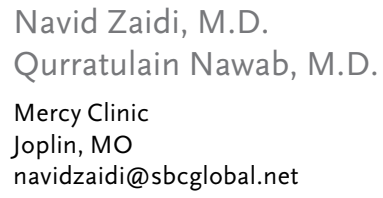

No potential conflict of interest relevant to this letter was reported.

1. Wenzel RP, Fowler AA III, Edmond MB. Antibiotic prevention of acute exacerbations of COPD. N Engl J Med 2012;367:340-7.

2. Tashkin DP, Celli B, Senn S, et al. A 4-year trial of tiotropium in chronic obstructive pulmonary disease. N Engl J Med 2008; 359:1543-54.

3. Calverly PMA, Rabe KF, Goehring UM, Kristiansen S, Fabbri LM, Martinez FJ. Roflumilast in symptomatic chronic obstructive pulmonary disease; two randomised clinical trials. Lancet 2009;374:685-94. [Erratum, Lancet 2010;376:1146.]

DOI: 10.1056/NEJMc1210335

TO THE EDITOR: Wenzel et al. describe adverse effects that may occur with yearlong use of azithromycin to prevent exacerbations of COPD. These adverse effects include hearing loss, a prolonged QTc interval associated with an increased risk of torsades de pointes, and drug-drug interactions. The authors also express concern about the development of bacterial resistance to azithromycin. To decrease the potential for harm, the authors suggest audiography and electrocardiography at the initial screening and every 3 months. The potential benefit is to reduce the frequency of COPD exacerbations from 1.83 to 1.48 per patient-year, and yearlong azithromycin treatment may result in fewer COPD-related hospitalizations. $^{1}$

Another approach is to give the patient azithromycin and instructions to begin treatment for 5 days if a worsening cough or change in sputum color or amount occurs. The therapeutic benefit of this approach is suggested by a recent study that showed that pulsed 5-day treatment with moxifloxacin at a dose of $400 \mathrm{mg}$ once a day every 8 weeks for 48 weeks was most effective in the subgroup of 323 patients with purulent sputum at baseline. ${ }^{2}$ Less frequent antibiotic use will decrease adverse effects, the need for monitoring, and expense.

\section{David Siegel, M.D., M.P.H.}

Department of Veterans Affairs Northern California Health Care System

Mather, CA

david.siegel@va.gov

No potential conflict of interest relevant to this letter was reported.

1. Albert RK, Connett J, Bailey WC, et al. Azithromycin for prevention of exacerbations of COPD. N Engl J Med 2011;365:68998. [Erratum, N Engl J Med 2012;366:1356.]

2. Sethi S, Jones PW, Theron MS, et al. Pulsed moxifloxacin for the prevention of exacerbations of chronic obstructive pulmonary disease: a randomized controlled trial. Respir Res 2010;11:10.

DOI: $10.1056 / N E J M c 1210335$

TO THE EDITOR: Wenzel et al. endorse the use of long-term prophylactic antibiotic therapy in certain patients with COPD. They support their recommendation with data from a trial by Albert et al. ${ }^{1}$ showing the "frequency of exacerbations was 1.48 per year in the azithromycin group versus 1.83 in the placebo group." However, although there was a modest improvement in the number of exacerbations, there was no significant difference in overall mortality between the groups. This finding raises an important question: Is it reasonable to endorse long-term prophylactic antibiotic therapy when there is no mortality benefit? It is clear that long-term use will hasten antibiotic resistance. The patient is, in effect, acting as culture medium to grow azithromycin-resistant organisms. Although the patient receives a modest benefit, the cost is going to be inflicted on society at large. In fact, the development of routine antibiotic resistance may even lead to an increased mortality in the population as a whole. Antibiotics are a communal resource, and the use of these agents on a long-term basis for reasons other than the prevention of death shows the selfish individualism of modern medicine and should not be taken lightly.

Dana G. Schwarz, M.D., Ph.D.

584 Morning Glory Dr.

Benicia, CA

dana.schwarz@gmail.com

No potential conflict of interest relevant to this letter was reported.

1. Albert RK, Connett J, Bailey WC, et al. Azithromycin for prevention of exacerbations of COPD. N Engl J Med 2011;365:68998. [Erratum, N Engl J Med 2012;366:1356.]

DOI: 10.1056/NEJMc1210335 
TO THE EDITOR: Wenzel et al. review the use of macrolide therapy for the prevention of COPD exacerbations. However, we have some concerns about the extrapolation of their conclusions worldwide. Different epidemiologic characteristics, rates of antibiotic resistance, and mechanisms of resistance among respiratory pathogens could be encountered in different countries.

The prevalence of macrolide-resistant Streptococcus pneumoniae, a major type of bacteria isolated in patients admitted for COPD exacerbation, is close to $96 \%$ in some Asian countries. ${ }^{1}$ Moreover, there are two common mechanisms of macrolide resistance in S. pneumoniae. The first mechanism involves the site where macrolides bind to the ribosome; this mechanism accounts for more than $90 \%$ of the macrolide-resistant isolate strains in Europe and confers high resistance (minimum inhibitory concentration, $\geq 64$ $\mu \mathrm{g}$ per milliliter). ${ }^{2}$ The second mechanism is caused by an efflux pump; this mechanism accounts for more than $65 \%$ of the macrolide-resistant isolate strains in the United States ${ }^{3}$ and confers a low level of macrolide resistance (minimum inhibitory concentration, $\geq 4 \mu \mathrm{g}$ per milliliter). The study conducted by Albert et al. ${ }^{4}$ was performed in the United States.

Before the use of azithromycin can be recommended worldwide, further studies should be performed in different geographic areas.

\section{Carolina Garcia-Vidal, M.D., Ph.D. \\ Diego Viasus, M.D., Ph.D. \\ Jordi Carratalà, M.D., Ph.D.}

Hospital Universitari de Bellvitge

Barcelona, Spain

carolgv75@hotmail.com

No potential conflict of interest relevant to this letter was reported.

1. Hsueh PR, Teng LJ, Lee LN, Yang PC, Ho SW, Luh KT. Extremely high incidence of macrolide and trimethoprim-sulfamethoxazole resistance among clinical isolates of Streptococcus pneumoniae in Taiwan. J Clin Microbiol 1999;37:897-901.

2. Reinert RR, Reinert S, van der Linden MY, Cil MY, Al-Lahham A, Appelbaum P. Antimicrobial susceptibility of Streptococcus pneumoniae in eight European countries from 2001 to 2003. Antimicrob Agents Chemother 2005;49:2903-13.

3. Brown SD, Farrell DJ, Morrissey I. Prevalence and molecular analysis of macrolide and fluoroquinolone resistance among isolates of Streptococcus pneumoniae collected during the 20002001 PROTEKT US Study. J Clin Microbiol 2004;42:4980-7.

4. Albert RK, Connett J, Bailey WC, et al. Azithromycin for prevention of exacerbations of COPD. N Engl J Med 2011;365:68998. [Erratum, N Engl J Med 2012;366:1356.]

DOI: 10.1056/NEJMc1210335
TO THE EDITOR: In their review of long-term azithromycin use for prevention of COPD exacerbations, Wenzel and coauthors acknowledge the risk of the development of infection with macrolide-resistant nontuberculous mycobacteria, and they recommend screening for mycobacteria before the initiation of azithromycin therapy and not administering this prophylaxis to patients with positive sputum cultures. The relationship between macrolides and nontuberculous mycobacteria may be more complex than described, however. In addition to the specific risk of macrolide resistance, ${ }^{1}$ retrospective studies involving patients with cystic fibrosis suggest that azithromycin may increase the risk of the development of infections with nontuberculous mycobacteria, ${ }^{2,3}$ and widespread use of long-term azithromycin may in part explain the alarming increase in the incidence of infections with nontuberculous mycobacteria observed in patients with cystic fibrosis. Experimental evidence suggests a paradoxical effect of azithromycin on nontuberculous mycobacteria, with bactericidal effects offset by reduced mycobacterial killing through inhibition of macrophage autophagy ${ }^{3}$ - a more complex picture than that depicted in Figure 1 of the article by Wenzel et al. As the authors note, the long-term adverse effects of macrolide use in patients with COPD remain unknown, but clinicians should remain alert to the possibility of the development of nontuberculous mycobacterial disease in patients during prolonged use of azithromycin prophylaxis.

Stephen J. Chapman, D.M.
Oxford Radcliffe Hospitals
Oxford, United Kingdom
schapman@well.ox.ac.uk
$\quad$ No potential conflict of interest relevant to this letter was re-
ported.
ported.

1. Griffith DE, Aksamit T, Brown-Elliott BA, et al. An official ATS/IDSA statement: diagnosis, treatment, and prevention of nontuberculous mycobacterial diseases. Am J Respir Crit Care Med 2007;175:367-416. [Erratum, Am J Respir Crit Care Med 2007;175:744-5.]

2. Levy I, Grisaru-Soen G, Lerner-Geva L, et al. Multicenter cross-sectional study of nontuberculous mycobacterial infections among cystic fibrosis patients, Israel. Emerg Infect Dis 2008; $14: 378-84$.

3. Renna M, Schaffner C, Brown K, et al. Azithromycin blocks autophagy and may predispose cystic fibrosis patients to mycobacterial infection. J Clin Invest 2011;121:3554-63.

DOI: $10.1056 /$ NEJMc1210335 
THE AUTHORS REPLY: Zaidi and Nawab argue that tiotropium or roflumilast could be initiated before considering azithromycin prophylaxis. Each suggestion has merit, but we offer some cautions. A recent meta-analysis revealed that patients receiving tiotropium had a modest reduction of 0.3 exacerbations per patient-year. ${ }^{1}$ Roflumilast was associated with a reduction in the rates of moderate-to-severe exacerbations: 1.14 per patient-year with roflumilast versus 1.37 with placebo. ${ }^{2}$ However, diarrhea (sometimes requiring hospitalization), nausea, and headache are common with roflumilast. Dose-limiting weight loss and depression may also occur.

Citing a study of pulsed moxifloxacin prophylaxis, Siegel argues for the preemptive use of azithromycin at the first sign of a possible exacerbation. Moxifloxacin was associated with a reduction in exacerbations by $19 \%$ but was not associated with improvement in lung function or quality-of-life score. ${ }^{3}$ Nevertheless, we do not think there are sufficient data to endorse the use of moxifloxacin or azithromycin preemptively.

Schwarz suggests that since no mortality benefit has been demonstrated, society at large could lose as a result of widespread antibiotic use in individual patients. His is a provocative medical and ethical focus on the principle of justice. Formal risk-benefit studies involving large numbers of patients with COPD would offer insights on morbidity, mortality, and costs of antibiotic prophylaxis. We would offer caution, however, with regard to a sole focus on mortality, because patients with COPD exacerbations have progressive decreases in lung function and quality of life and increases in disability. They often live and eventually die with marked air hunger.

We agree with Garcia-Vidal et al. that findings in studies of azithromycin in one part of the world may not apply equally in all countries. Since azithromycin may have important antiinflammatory activity, however, it may be premature to focus on varying mechanisms of antibacterial resistance in different regions globally.
Chapman focuses on the emergence of infections with nontuberculous mycobacteria among patients with cystic fibrosis who have received prophylactic azithromycin. He warns that a similar emergence could happen among patients with COPD as well. In the study involving patients with cystic fibrosis from Israel cited by Chapman, although there were differences in a univariate analysis relating prior azithromycin use to infections with nontuberculous mycobacteria, the multivariate analysis showed none the adjusted odds ratio was 1.0. ${ }^{4}$ In the study from the United Kingdom, at a single center a correlation over time was noted between the number of patients with cystic fibrosis receiving long-term azithromycin therapy and the number with new nontuberculous mycobacteria cultures. ${ }^{5}$ Causality cannot yet be ascribed, however. We appreciate the concern aroused but conclude that screening for colonization with nontuberculous mycobacteria more than once a year is currently unwarranted.

Richard P. Wenzel, M.D.

Alpha A. Fowler III, M.D.

Michael B. Edmond, M.D., M.P.H.

Virginia Commonwealth University Medical Center

Richmond, VA

rwenzel@mcvh-vcu.edu

Since publication of their article, the authors report no further potential conflict of interest.

1. Van den Bruel A, Gailly J, Neyt M. Does tiotropium lower exacerbation and hospitalization frequency in COPD patients: results of a meta-analysis. BMC Pulm Med 2010;10:50.

2. Calverley PMA, Rube KF, Goehring UM, Kristiansen S, Fabbri LM Martinez FJ. Roflumilast in symptomatic chronic obstructive pulmonary disease: two randomised clinical trials. Lancet 2009;374:685-94. [Erratum, Lancet 2010;376:1146.]

3. Sethi S, Jones PW, Theron MS, et al. Pulsed moxifloxacin for the prevention of exacerbations of chronic obstructive pulmonary disease: a randomized controlled trial. Respir Res 2010;11:10. [Erratum, Respir Res 2010;11:88.]

4. Levy I, Grisaru-Soen G, Lerner-Geva L, et al. Multicenter cross-sectional study of nontuberculous mycobacterial infections among cystic fibrosis patients, Israel. Emerg Infect Dis 2008;14:378-84.

5. Renna M, Schaffner C, Brown K, et al. Azithromycin blocks autophagy and may predispose cystic fibrosis patients to mycobacterial infection. J Clin Invest 2011;121:3554-63.

DOI: 10.1056/NEJMc1210335

\section{A Sea-Urchin Spine Chilling Remedy}

TO THE EDITOR: One of the potential consequences of recreational diving and surfing is injury caused by a sea-urchin spine. Injury can oc- cur as people enter or exit rocky shorelines and is felt immediately as a sharp, localized pain, which is exacerbated by the application of pressure. If 